\title{
STABILIZATION PROBLEM FOR NONHOLONOMIC CONTROL SYSTEMS
}

\author{
L. RIFFORD* \\ Laboratoire J.A. Dieudonné, Université de Nice-Sophia Antipolis, \\ Parc Valrose, 06108 Nice Cedex 02, France \\ *E-mail: rifford@math.unice.fr
}

\begin{abstract}
We present the stabilization problem in the context of nonholonomic control systems and nonholonomic distributions. Then, we introduce the notions of smooth repulsive stabilizing feedbacks and sections, and review recent results on the existence of such objects.
\end{abstract}

Keywords: Stabilization problem

\section{Introduction}

Throughout this paper, $M$ denotes a smooth connected manifold of dimension $n$ and $\bar{x}$ a point of $M$.

\subsection{Stabilization of nonholonomic control systems}

Let $f_{1}, \cdots, f_{m}$ be a family of $m$ smooth vector fields on $M$. We say that the control system defined as,

$$
\dot{x}=\sum_{i=1}^{m} u_{i} f_{i}(x)
$$

is nonholonomic on $M$ (also called totally nonholonomic in Ref. 1) if the following property is satisfied:

$$
\text { Lie }\left\{f_{1}, \cdots, f_{m}\right\}(x)=T_{x} M, \quad \forall x \in M .
$$

Recall that, for every $x \in M$ and every control $u(\cdot) \in L^{1}\left([0, \infty) ; \mathbb{R}^{m}\right)$, there is a unique maximal solution $x(\cdot)=x(\cdot ; u, x):\left[0, T_{u}\right) \rightarrow M\left(\right.$ with $\left.T_{u}>0\right)$ to the Cauchy problem

$$
\dot{x}(t)=\sum_{i=1}^{m} u_{i}(t) f_{i}(x(t)), \quad \text { for a.e. } \quad t \in\left[0, T_{u}\right), \quad x(0)=x .
$$


As the next result shows, any nonholonomic control system is controllable on $M$ (see Refs. 2 or 3 ).

Theorem 1.1 (Chow-Rashevsky Theorem). Let (1) be a nonholonomic control system on $M$. Then, for every pair $x, y \in M$, there exists a smooth control $u(\cdot):[0,1] \rightarrow \mathbb{R}^{m}$ such that $x(1 ; u, x)=y$.

Before presenting the stabilization problem, we need to recall the notion of globally asymptotically stable dynamical system. Let $X$ be a smooth vector field on $M$, the dynamical system $\dot{x}=X(x)$ is said to be globally asymptotically stable at the point $\bar{x}$ (abreviated $\mathrm{GAS}_{\bar{x}}$ in the sequel), if the two following properties are satisfied:

(i) Lyapunov stability: for every neighborhood $\mathcal{V}$ of $\bar{x}$, there exists a neighborhood $\mathcal{W}$ of $\bar{x}$ such that, for every $x \in \mathcal{W}$, the solution of $\dot{x}(t)=X(x(t)), x(0)=x$, satisfies $x(t) \in \mathcal{V}$, for every $t \geq 0$.

(ii) Attractivity: for every $x \in M$, the solution of $\dot{x}(t)=X(x(t)), x(0)=x$, tends to $\bar{x}$ as $t$ tends to $+\infty$.

The purpose of the stabilization problem is the following:

Let (1) be a given nonholonomic control system, does there exist a smooth ${ }^{\mathrm{a}}$ mapping $k: M \rightarrow \mathbb{R}^{m}$ (called stabilizing feedback) such that the dynamical system (called closed-loop system) defined as,

$$
\dot{x}=\sum_{i=1}^{m} k(x)_{i} f_{i}(x)
$$

is $\operatorname{GAS}_{\bar{x}}$ ?

\subsection{Stabilization problem for nonholonomic distributions}

Let $\Delta$ be a (totally) nonholonomic distribution of rank $m \leq n$ on $M$. This means that for every $x \in M$, there is a neighborhood $\mathcal{V}_{x}$ of $x$ in $M$ and a $m$-tuple $\left(f_{1}^{x}, \cdots, f_{m}^{x}\right)$ of smooth vector fields on $\mathcal{V}_{x}$ such that

$$
\Delta(y)=\operatorname{Span}\left\{f_{1}^{x}(y), \cdots, f_{m}^{x}(y)\right\}, \quad \forall y \in \mathcal{V}_{x},
$$

and moreover,

$$
\text { Lie }\left\{f_{1}^{x}, \cdots, f_{m}^{x}\right\}(y)=T_{y} M, \quad \forall y \in M
$$

\footnotetext{
${ }^{\mathrm{a}}$ We restrict here our attention to smooth stabilizing feedbacks. In fact, it can be shown that if the control system (1) admits a continuous stabilizing feedback then this feedback can be regularized into a smooth stabilizing feedback.
} 
We call horizontal path between $x$ to $y$, any absolutely continuous curve $\gamma(\cdot):[0,1] \rightarrow M$ with $\gamma(0)=x, \gamma(1)=y$ which satisfies

$$
\dot{\gamma}(t) \in \Delta(\gamma(t)), \quad \text { for a.e. } t \in[0,1] .
$$

In the context of nonholonomic distribution, the Chow-Rashevsky Theorem takes the following form.

Theorem 1.2. Let $\Delta$ be a nonholonomic distribution on $M$. Then, any two points of $M$ can be joined by a smooth horizontal path.

The stabilization problem for nonholonomic distributions consists in finding, if possible, a smooth stabilizing section of $\Delta$ at $\bar{x}$, that is, a smooth vector field $X$ on $M$ satisfying $X(x) \in \Delta(x)$ for every $x \in M$ and such that the dynamical system $\dot{x}=X(x)$ is $\operatorname{GAS}_{\bar{x}}$.

\subsection{Two obstructions}

Given a nonholonomic control system (resp. a nonholonomic distribution), there are two obstructions to the existence of stabilizing feedbacks (resp. stabilizing sections). The first one is global while the second one is purely local.

(i) Global obstruction: If fact, if the manifold $M$ admits a smooth dynamical system which is $\mathrm{GAS}_{\bar{x}}$, then it must be homeomorphic to $\mathbb{R}^{n}$ (see Ref. 4 for further details).

(ii) Local obstruction: Since this obstruction is local, we can assume that we work in $\mathbb{R}^{n}$, that is, in an open neighborhood $\mathcal{U} \subset \mathbb{R}^{n}$ of $\bar{x}$. If there is a smooth vector field $X$ on $\mathcal{U}$ which is locally asymptotically stable at $\bar{x}$, then for all $\epsilon$ small enough,

$$
\exists \delta>0 \text { such that } \delta B \subset X(\bar{x}+\epsilon B),
$$

where $B$ denotes the open unit ball in $\mathbb{R}^{n}$ (see Ref. 4 for further details). In consequence, if there is a smooth feedback $k: \mathcal{U} \rightarrow \mathbb{R}^{m}$ such that the closed-loop system (2) is locally asymptotically stable at $\bar{x}$, then the result above applies to the dynamics $X(x)=\sum_{i=1}^{m} k(x)_{i} f_{i}(x)$. Thus, for all $\epsilon$ small enough,

$$
\exists \delta>0 \text { such that } \delta B \subset\left\{\sum_{i=1}^{m} u_{i} f_{i}(\bar{x}+\epsilon B) \mid u \in \mathbb{R}^{m}\right\},
$$

which is the Brockett necessary condition (see Ref. 5). In particular, we deduce that any distribution $\Delta$ of rank $m<n$ cannot admit a smooth locally stabilizing section at $\bar{x}$. 
The obstructions above make it impossible to prove the existence of smooth stabilizing feedbacks (resp. sections) for nonholonomic control systems (resp. distributions). Actually, they motivate the design of new kinds of stabilizing feedbacks. The main contributions in that direction have been: Sussmann,${ }^{6}$ Coron, ${ }^{7}$ Clarke, Ledyaev, Sontag and Subbotin, ${ }^{8}$ and Ancona and Bressan. ${ }^{9}$ The aim of the present paper is to highlight the notion of smooth repulsive stabilizing feedbacks (or sections) and to show that it permits to stabilize most of the nonholonomic control systems (or distributions).

\section{Examples}

\subsection{The Nonholonomic integrator}

Define in $\mathbb{R}^{3}$ the two smooth vector fields $f_{1}, f_{2}$ by,

$$
f_{1}=\frac{\partial}{\partial x_{1}}+x_{2} \frac{\partial}{\partial x_{3}} \quad \text { and } \quad f_{2}=\frac{\partial}{\partial x_{2}}-x_{1} \frac{\partial}{\partial x_{3}} .
$$

Note that at any point $x \in \mathbb{R}^{3}$, the three vectors $f_{1}(x), f_{2}(x),\left[f_{1}, f_{2}\right](x)$ are linearly independent. Hence the associated control system

$$
\dot{x}=u_{1} f_{1}(x)+u_{2} f_{2}(x),
$$

is nonholonomic on $\mathbb{R}^{3}$. As it is well-known, the nonholonomic integrator can be stabilized at the origin by a feedback which is smooth outside the vertical line. Denote by $\mathcal{S}$ the vertical line of equation $x_{1}=x_{2}=0$ in $\mathbb{R}^{3}$. Using an adapted control-Lyapunov function (see Refs. 10 or 11 for further details), we can construct a mapping $k=\left(k_{1}, k_{2}\right): \mathbb{R}^{3} \mapsto \mathbb{R}^{2}$ in such a way that the following properties are satisfied:

(i) The mapping $k$ is locally bounded and smooth on $M \backslash \mathcal{S}$.

(ii) The set $\mathbb{R}^{3} \backslash \mathcal{S}$ is invariant with respect to the dynamical system

$$
\dot{x}=k_{1}(x) f_{1}+k_{2}(x) f_{2}(x),
$$

and steers asymptotically all its trajectories to the origin.

In fact, the feedback above can be extended to the whole space $\mathbb{R}^{3}$. In this way, we can construct a feedback which is smooth outside $\mathcal{S}$, discontinuous at the points of $\mathcal{S}$, and for which the closed-loop system is $\mathrm{GAS}_{\bar{x}}$ in the sense of Carathéodory (see below). Such a feedback is an example of what we call a smooth repulsive stabilizing feedback. 


\subsection{The Riemannian case}

Assume through this paragraph that the nonholonomic distribution $\Delta$ is given by $\Delta(x)=T_{x} M$ for every $x \in M$, our aim is to show how to construct a stabilizing section for $\Delta$. For that, consider a smooth and complete Riemannian metric $g$ on $M$ and denote by $d_{g}$ the Riemannian distance associated with $g$. We recall that, for any $x, y \in M$, the Riemannian distance between $x$ and $y$ is defined as

$$
d_{g}(x, y)=\inf \left\{\operatorname{length}_{g}(\gamma(\cdot))\right\}, \quad \forall x, y \in M,
$$

where the infimum is taken over all the $C^{1}$ paths $\gamma(\cdot):[0,1] \rightarrow M$ joining $x$ to $y$, and where

$$
\operatorname{length}_{g}(\gamma(\cdot))=\int_{0}^{1} \sqrt{g_{\gamma(t)}(\dot{\gamma}(t), \dot{\gamma}(t))} d t .
$$

Let $V_{g}: M \rightarrow \mathbb{R}$ be the function defined by

$$
V_{g}(x)=d_{g}(\bar{x}, x)^{2}, \quad \forall x \in M
$$

It is easy to show that $V_{g}$ is Lipschitz continuous on $M$ and smooth outside the set $\mathcal{S}$ defined as the cut-locus ${ }^{\mathrm{b}}$ from the point $\bar{x}$. Define the vector field $X$ on $M$ by

$$
X(x)=-\nabla_{g} V_{g}(x), \quad \forall x \in M \backslash \mathcal{S},
$$

where $\nabla_{g} V_{g}(x)$ denotes the gradient of $V_{g}$ at $x$ with respect to the metric $g$. By construction, any trajectory of $\dot{x}(t)=X(x(t))$ tends to $\bar{x}$ as $t \rightarrow \infty$ and satisfies the following property:

$$
\forall t \geq 0, \quad x(t) \notin \mathcal{S} .
$$

In fact, $X$ can be extended into a global section of $\Delta$ on $M$ which is smooth outside the cut-locus from $\bar{x}$, discontinuous at the points of $\mathcal{S}$ and whose the associated dynamics drives all its Carathéodory trajectories asymtptically to $\bar{x}$. Such a stabilizing section corresponds to what we call a smooth repulsive stabilizing section of $\Delta$ on $M$.

\section{Smooth repulsive stabilization}

Our aim is now to make precise the notions of smooth repulsive stabilizing feedbacks or sections, and to show what kind of results we are able to prove.

\footnotetext{
b The cut-locus from $\bar{x}$ is defined as the closure of the set where $V_{g}$ is not differentiable. We refer the reader to Ref. 12 for a detail study of the distance function and the cut-locus from $\bar{x}$.
} 


\section{1. $S R S_{\bar{x}, \mathcal{S}}$ vector fields}

Let $\mathcal{S}$ be a closed subset of $M$ and $X$ be a smooth vector field on $M$. The dynamical system $\dot{x}=X(x)$ is said to be smooth repulsive globally asymptotically stable at $\bar{x}$ with respect to $\mathcal{S}$ (denoted in short $\mathrm{SRS}_{\bar{x}, \mathcal{S}}$ ) if the following properties are satisfied:

(i) The vector field $X$ is locally bounded on $M$ and smooth on $M \backslash \mathcal{S}$.

(ii) The dynamical system $\dot{x}=X(x)$ is globally asymptotically stable at $\bar{x}$ in the sense of Carathéodory, namely, for every $x \in M$, there exists a solution of

$$
\dot{x}(t)=X(x(t)), \quad \text { for almost every } t \in[0, \infty), \quad x(0)=x,
$$

and, for every $x \in M$, every solution of (3) (called Carathéodory solution of $\dot{x}=X(x))$ on $[0, \infty)$ tends to $\bar{x}$ as $t$ tends to $\infty$. Moreover, for every neighborhood $\mathcal{V}$ of $\bar{x}$, there exists a neighborhood $\mathcal{W}$ of $\bar{x}$ such that, for $x \in \mathcal{W}$, the solutions of (3) satisfy $x(t) \in \mathcal{V}$, for every $t \geq 0$.

(iii) For every $x \in M$, the solutions of (3) satisfy $x(t) \notin \mathcal{S}$, for every $t>0$.

Given the nonholonomic control system (1), we shall say that a mapping $k: M \rightarrow \mathbb{R}^{m}$ is a smooth repulsive stabilizing feedback at $\bar{x}$ (denoted in short $\mathrm{SRS}_{\bar{x}}$ feedback) for (1), if it is locally bounded on $M$ and if there is a closed set $\mathcal{S} \subset M$ such that $k$ is smooth on $M \backslash \mathcal{S}$ and such that its associated closed-loop system is $\mathrm{SRS}_{\bar{x}, \mathcal{S}}$. In the same way, given a nonholonomic distribution $\Delta$ and a vector field $X$ on $M$, we shall say that $X$ is a smooth repulsive stabilizing section at $\bar{x}$ (denoted in short $\operatorname{SRS}_{\bar{x}}$ section) for $\Delta$, if $X$ is a section of $\Delta$ on $M$ and if the dynamical system $\dot{x}=X(x)$ is $\operatorname{SRS}_{\bar{x}, \mathcal{S}}$ for some closed set $\mathcal{S} \subset M$.

\subsection{Existence results of $S R S_{\bar{x}}$ feedbacks}

Using a technique of cancellation of the bifurcation points of a "discontinuous" stabilizing feedback, we proved in Ref. 13 the following result:

Theorem 3.1. If $M$ has dimension two, any nonholonomic control system of the form (1) admits a $S R S_{\bar{x}}$ feedback.

Using the classical method of local approximation of a nonholonomic control systems by an homogeneous one together with the technique of cancellation developed on surfaces, we were able in Ref. 14 to demonstrate the following result: 
Theorem 3.2. If $M$ has dimension three, any nonholonomic control system of the form (1) admits a SRS $S_{\bar{x}}$ feedback defined on a neighborhood of $\bar{x}$.

We refer the interested reader to Refs. 13 and 14 for more details on these results.

\subsection{Existence results of $S R S_{\bar{x}}$ sections}

The method presented above in the Riemannian case can also be developed in the sub-Riemannian setting; we need for that to introduce material of sub-Riemannian geometry. Let $\Delta$ be a nonholonomic distribution of rank $m \leq n$ on $M$, the set of horizontal paths $\gamma(\cdot):[0,1] \rightarrow M$ such that $\gamma(0)=\bar{x}$, denoted by $\Omega_{\Delta}(\bar{x})$, endowed with the $W^{1,1}$-topology, inherits of a Banach manifold structure (see Ref. 15 for further details). The end-point mapping from $\bar{x}$ is the mapping $E_{\bar{x}}: \Omega_{\Delta}(\bar{x}) \rightarrow M$ defined by

$$
E_{\bar{x}}(\gamma(\cdot))=\gamma(1), \quad \forall \gamma(\cdot) \in \Omega_{\Delta}(\bar{x}) ;
$$

it is a smooth mapping on $\Omega_{\Delta}(\bar{x})$. A path $\gamma(\cdot)$ is said to be singular if it is horizontal and if it is a critical point of the end-point mapping $E_{\bar{x}}$, that is, if the differential of $E_{\bar{x}}$ at $\gamma(\cdot)$ is not a submersion. Let $g$ be a smooth Riemannian metric on $M$, the sub-Riemannian distance $d_{S R}(x, y)$ between two points $x, y$ of $M$ is defined by

$$
d_{S R}(x, y)=\inf \left\{\operatorname{length}_{g}(\gamma(\cdot)) \mid \gamma(\cdot) \in \Omega_{\Delta}(\bar{x})\right\} .
$$

According to the Chow-Rashevsky Theorem, since $\Delta$ is nonholonomic on $M$, the sub-Riemannian distance is well-defined and continuous on $M \times$ $M$. Moreover, if the manifold $M$ is a complete metric space ${ }^{\mathrm{c}}$ for the subRiamannian distance $d_{S R}$, then, since $M$ is connected, for every pair $x, y$ of points of $M$ there exists an horizontal path $\gamma(\cdot):[0,1] \rightarrow M$ joining $x$ to $y$ such that

$$
d_{S R}(x, y)=\operatorname{length}_{g}(\gamma(\cdot)) .
$$

Such a horizontal path is said to be minimizing. The following result has been obtained recently with Trélat (see Ref. 15).

Theorem 3.3. Let $\Delta$ be a smooth nonholonomic distribution of rank $m \leq$ $n$ on $M$. Assume that there exists a smooth Riemannian metric $g$ on $\Delta$ for

\footnotetext{
$\overline{{ }^{\mathrm{c}} \text { Note that, since the distribution }} \Delta$ is nonholonomic on $M$, the topology defined by the sub-Riemannian distance $d_{S R}$ coincides with the original topology of $M$ (see Refs 2 or 3).
} 
which $M$ is complete and no nontrivial singular path is minimizing. Then, there exist a section $X$ of $\Delta$ on $M$, and a closed nonempty subset $\mathcal{S}$ of $M$, of Hausdorff dimension lower than or equal to $n-1$, such that $X$ is $S R S_{\bar{x}, \mathcal{S}}$.

Note that if $m=n$, then obviously there exists no singular path (it is the Riemannian case). In fact, the main assumption of Theorem 3.3 (the absence of nontrivial singular minimizing paths) is automatically satisfied for a large class of sub-Riemannian structures such as the fat distributions or the medium-fat distributions associated with a generic metric (we refer the reader to Ref. 15 for further details). Here, we just want to emphasize the fact that the main assumption of Theorem 3.3 is satisfied generically for distributions with rank greater than two.

Let $m \geq 3$ be a positive integer and $\mathcal{G}_{m}$ be the set of pairs $(\Delta, g)$, where $\Delta$ is a rank $m$ distribution on $M$ and $g$ is a smooth Riemannian metric on $\Delta$, endowed with the Whitney $C^{\infty}$ topology. It is shown in Ref. 16 that there exists an open dense subset $W_{m}$ of $\mathcal{G}_{m}$ such that every element of $W_{m}$ does not admit nontrivial minimizing singular paths. This means that, for $m \geq 3$, generically, the main assumption of Theorem 3.1 is satisfied. Therefore, as a by-product of the Chitour-Jean-Trélat Theorem, we have the following result:

Corollary 3.1. A generic nonholonomic distribution of rank $\geq 3$ admits a $S R S_{\bar{x}}$ section.

We notice that in Ref. 15, we are able to remove, in the compact and orientable three-dimension case, the assumption on the absence of singular minimizing paths. We refer the interested reader to Ref. 15 for further details on that result.

\subsection{A Nonholonomic dream}

In view of the results presented here, one might hope that the following conjecture is true.

Conjecture. Any nonholonomic control system admits a SRS feedback.

\section{References}

1. A. A. Agrachev and Y. L. Sachkov. Control theory from the geometric viewpoint. Encyclopaedia of Mathematical Sciences, 87. Springer-Verlag, Berlin, 2004. 
2. A. Bellaïche. The tangent space in sub-Riemannian geometry. in SubRiemannian Geometry, Birkhäuser, 1-78, 1996.

3. R. Montgomery. A tour of subriemannian geometries, their geodesics and applications. Mathematical Surveys and Monographs, Vol. 91. American Mathematical Society, Providence, RI, 2002.

4. L. Rifford. The Stabilization Problem: AGAS and SRS Feedbacks. In Optimal Control, Stabilization, and Nonsmooth Analysis, Lectures Notes in Control and Information Sciences, 301, Springer-Verlag, Heidelberg (2004), 173-184.

5. R. W. Brockett. Asymptotic stability and feedback stabilization. Differential geometric control theory, R. W. Brockett, R. S. Millman and H. J. Sussmann, ed., Boston, Birkhäuser, 181-191, 1983.

6. H. J. Sussmann. Subanalytic sets and feedback control. J. Differential Equations, 31(1):31-52, 1979.

7. J.-M. Coron. Global asymptotic stabilization for controllable systems without drift. Math. Control Signals Systems, 5(3):295-312, 1992.

8. F. H. Clarke, Yu. S. Ledyaev, E.D. Sontag, and A.I. Subbotin. Asymptotic controllability implies feedback stabilization. I.E.E.E. Trans. Aut. Control, 42:1394-1407, 1997.

9. F. Ancona and A. Bressan. Patchy vector fields and asymptotic stabilization. ESAIM Control Optim. Calc. Var., 4:445-471, 1999.

10. L. Rifford. Problèmes de stabilisation en théorie du contrôle. PhD thesis, Université Lyon I, 2000.

11. Yu. S. Ledyaev and L. Rifford. Robust stabilization of the nonholonomic integrator. Unpublished.

12. T. Sakai. Riemannian geometry. Translations of Mathematical Monographs, Vol. 149. American Mathematical Society, Providence, RI, 1996.

13. L. Rifford. The stabilization problem on surfaces. Rend. Semin. Mat. Torino 64(1):55-61, 2006.

14. L. Rifford. On the existence of local smooth repulsive stabilizing feedbacks in dimension three. J. Differential Equations, 226(2):429-500, 2006.

15. L. Rifford and E. Trélat. On the stabilization problem for nonholonomic distributions. Preprint, 2006.

16. Y. Chitour, F. Jean and E. Trélat. Genericity results for singular curves. J. Diff. Geom. 73(1):45-73, 2006. 